\title{
Percepción de los Beneficios de la Tenencia de Animales de Compañía en Personas con Orientación Sexual Homoerótica yTransexuales
}

\author{
Perception of the Benefits of the Ownership of Companion Animals in People \\ with Homoerotic Sexual Orientation and TransseXuals
}
Beatriz Hugues H. ${ }^{1,2}$, Aimee Álvarez A. ${ }^{1}$, Lizet Castelo E.C. ${ }^{1}$, Loraine Ledón L. ${ }^{1}$, Madelín Mendoza T. ${ }^{1}$, Emma Domínguez A. ${ }^{1}$

\section{Resumen}

\begin{abstract}
El objetivo del estudio fue conocer la percepción de personas de ambos sexos con orientación sexual homoerótica $(\mathrm{OSH})(\mathrm{n}=40)$ y de transexuales $(\mathrm{n}=20)$ con respecto a la tenencia de animales de compañía y su relación con la satisfacción personal y los beneficios sobre la salud psicoemocional. Se realizó una encuesta con base a un cuestionario autoadministrado. Se constató que el $83 \%$ de las personas con OSH y el $85 \%$ de los transexuales estaban muy satisfechos por convivir con animales de compañía. Para las personas con OSH, el $98 \%$ cuidaba más de su salud y al 93\% le proporcionaba compañía, mientras que para los transexuales, al $95 \%$ le proporcionaba bienestar y el $90 \%$ cuidaba más de su salud. En relación a los beneficios sobre la salud psicoemocional, el 83\% de las personas con OSH y el $90 \%$ de los transexuales aludieron que les resultaba muy beneficioso. Se concluye que los grupos estudiados percibían que la tenencia de animales de compañía les ejerció efectos beneficiosos para su satisfacción personal y salud emocional.
\end{abstract}

Palabras clave: animales de compañía; orientación sexual homoerótica; transexual

\section{Abstract}

The aim of this study was to become acquainted of the perception of persons with homoerotic sexual preference (HSP) $(n=40)$ and of transsexuals $(n=20)$ regarding pet ownership and its relationship with personal satisfaction and benefits on psicoemotional health. A survey using a questionnaire was conducted. The results showed that $83 \%$ of the persons with HSP and $85 \%$ of transsexuals were very satisfied with owning pets. Also, $98 \%$ SHO persons took more care of themselves and $93 \%$ of the transsexuals felt accompanied. In relation to the psicoemotional health $83 \%$ of HSP persons and $90 \%$ of

\footnotetext{
${ }^{1}$ Instituto Nacional de Endocrinología (INEN), La Habana, Cuba

${ }^{2}$ E-mail: bettymig@infomed.sld.cu
}

Recibido: 6 de junio de 2014

Aceptado para publicación: 24 de octubre de 2014 
transsexuals considered highly benefitial. It is concluded that the studied groups perceived personal satisfaction and better emotional health due to pet ownership.

Key words: pets; homoerotic sexual preference; transsexuals

\section{INTRODUCCIÓN}

La tenencia de los animales de compañía contribuye al bienestar de la persona a su cargo y de quienes se relacionan con ellos en los hogares. Se ha demostrado que mejoran la salud mental y la calidad de vida humana al reducir el estrés, promover la relajación, recreación y distracción, y aliviar la depresión, temores, angustias, ansiedad y sentimientos de soledad (Gómez et al., 2007). McConnell et al. (2011) demostraron que las personas que convivían con animales de compañía eran más felices, tenían mejor salud y estaban mejor ajustados psicológicamente que aquellas que no los tenían.

Los animales de compañía promueven la socialización entre personas desconocidas, permitiendo que se vuelven más solidarias. Asimismo, facilitan la comunicación entre generaciones, por ser un foco de interés común, tanto en el seno de la familia como fuera de ella (Jofré, 2005).

Por otro lado, en las personas con orientación sexual homoerótica (OSH) se presentan manifestaciones y actitudes de rechazo social y familiar, intolerancia, discriminación, censuras y fobias (homofobia, transfobia); factores que, en muchos casos, obligan a tomar doble conducta, con riesgos importantes desde los puntos de vista biológico, psicológico y social, tanto para el individuo como para su familia, lo cual ha sido demostrado en Cuba (Palú et al., 2010) y en otros lugares (Scourfield et al., 2008).

La transexualidad es un término creado por las ciencias médicas para designar a aquellas personas que, por lo general, desde su infancia temprana y a lo largo de su vida, manifiestan un indisoluble sentimiento de pertenecer a un género (femenino o masculino) que no se corresponde con el sexo asignado al nacer, según la morfología de sus genitales (Castro, 2008). Las personas transexuales expresan el sufrimiento constante que les produce la insatisfacción de sentirse atrapados(as) en un cuerpo con el cual no se identifican.

Teniendo en cuenta los efectos positivos que sobre la salud humana ejerce la convivencia con los animales de compañía, sobre todo, los efectos psicosemocionales y socializadores, es posible considerar que las personas de OSH y los transexuales pudieran beneficiarse al compartir sus vidas con animales de compañía. Sobre estas bases se planteó el presente estudio, con el objetivo de conocer la percepción que tienen personas de orientación sexual homoerótica y transexuales, que atienden a sus animales en una clínica veterinaria, con respecto a los beneficios de la tenencia de animales de compañía y su relación con la satisfacción personal y los beneficios que les proporciona en su salud psicoemocional.

\section{Materiales y Métodos}

Se realizó un estudio exploratorio, descriptivo, transversal, utilizando metodología cuantitativa de investigación, durante los meses de septiembre de 2011 a mayo de 2012.

La población objeto de estudio quedó conformada por 40 personas de orientación sexual homoerótica (30 varones y 10 mujeres) y 20 transexuales (originalmente del sexo 
Cuadro 1. Datos demográficos (\%) de las personas con orientación sexual homoerótica y que tienen animales de compañía

\begin{tabular}{llccc}
\hline \multirow{2}{*}{ Variable } & & \multicolumn{2}{c}{ Género } & Total \\
\cline { 3 - 4 } & $20-29$ & $\begin{array}{c}\text { Varones } \\
(\mathrm{n}=30)\end{array}$ & $\begin{array}{c}\text { Mujeres } \\
(\mathrm{n}=10)\end{array}$ & $(\mathrm{n}=40)$ \\
\hline Edad (años) & $30-39$ & 17 & - & 12 \\
& $40-49$ & 27 & 50 & 48 \\
& 50 o más & 10 & 30 & 28 \\
Ocupación & Estudiantes & 13 & 20 & 12 \\
& Trabajadores & - & 30 & 10 \\
Acuden a la consulta & Sin vínculo laboral & 87 & 70 & 82 \\
& Solos & 27 & 40 & 30 \\
Conviven con otras & En pareja & 73 & 60 & 70 \\
personas & Sí & 89 & 70 & 85 \\
& No & 11 & 30 & 15 \\
\hline
\end{tabular}

masculino que se identificaron sexual y genéricamente como mujeres). Estas personas asistieron con sus animales de compañía a solicitar los servicios de la consulta de oftalmología de la principal clínica de animales menores de La Habana «José Luis Callejas».

El conocimiento sobre la preferencia e identidad sexual y de género de estas personas fue el resultado de su autorreferencia espontánea. Como criterios de inclusión se consideraron a este tipo de personas, mayores de 18 años, y que tuvieran animales de compañía, y como criterios de exclusión a estas personas pero con condiciones físicas o de salud mental que les dificultaban la lectura y comprensión del cuestionario del estudio.

Se trabajó con un cuestionario sobre tenencia de animales de compañía de 10 ítems, en el cual se incluyeron los siguientes aspectos:

- Datos demográficos de las personas: sexo, edad y ocupación. Se registraba si acudían solas o acompañadas por sus pa- rejas a la consulta, y si vivían solas o con otras personas.

- Satisfacción personal sobre la tenencia de animales de compañía: Valoración de la satisfacción que siente la persona al estar en contacto directo con un animal de compañía, así como el bienestar que le produce esta interrelación. Se exploró sobre la especie de animal de compañía que poseían y el tiempo de convivencia con ellos; además, se indagó sobre el animal preferido. Los encuestados podían seleccionar más de una respuesta.

- Beneficios sobre la salud psicoemocional: Valoración de los posibles efectos beneficiosos que ejerce la tenencia de animales de compañía, relacionados con los aspectos de la salud psicoemocional y la calidad de vida de las personas a su cargo.

La participación de los sujetos en el estudio fue voluntaria y anónima. A cada persona que cumplió con los criterios de inclusión se le solicitó su participación, previa in- 
Cuadro 2. Datos demográficos de las personas transexuales y que tienen animales de compañía

\begin{tabular}{cc}
\hline Variable & $\begin{array}{c}\text { Frecuencia } \\
(\%)\end{array}$ \\
\hline Edad (años) & \\
$20-29$ & 40.0 \\
$30-39$ & 60.0 \\
$40-49$ & - \\
50 o más & - \\
Ocupación & - \\
Estudiantes & 35 \\
Trabajadores & 65 \\
Sin vínculo & \\
laboral & 20 \\
Acuden a la consulta & 80 \\
Solos & \\
En pareja & \\
Conviven con otras & 75 \\
personas & 25 \\
Sí & \\
No &
\end{tabular}

formación de las características del estudio. $\mathrm{Al}$ consentir su participación se le brindó el cuestionario y la información recogida fue manejada de forma confidencial. No se recolectaron datos de identidad personal y los datos obtenidos fueron codificados y analizados mediante estadística descriptiva en números enteros.

\section{Resultados y Discusión}

\section{Datos Demográficos}

Los datos demográficos de las personas con OSH se presentan en el Cuadro 1. El 75\% de personas de este grupo que llevaban a sus animales al servicio oftalmológico eran varones (30/40). En relación con el grupo etario, tanto en mujeres como en varones, fue más frecuente la presencia de personas entre 30 y 39 años (48\%). Asimismo, era más común los que tenían vínculo laboral $(82 \%)$ y convivían con otras personas $(85 \%)$.

La mayoría asistía a las consultas acompañado de sus parejas (70\%, Cuadro 1). Sin necesidad de preguntar, estas personas declaraban que el animal constituía su hijo(a) o alguien muy querido, y que se hiciera todo lo posible por recuperar su problema de salud.

El grupo de transexuales (Cuadro 2) estuvo constituido íntegramente por personas más jóvenes; entre los 20 y 39 años. El 65\% estaba vinculado laboralmente, el $80 \%$ acudía con su pareja a la consulta y también expresaba de forma libre y espontánea el significado que tenía para ellos la convivencia con estos animales.

\section{Satisfacción Personal}

La tenencia de animales de compañía, según especie, se muestra en los cuadros 3 y 4. El perro fue el animal mayoritario (60\% en OSH y $45 \%$ en transexuales), seguido por los gatos $(17 \%$ en OSH y $30 \%$ en transexuales) y aves ornamentales (12\% en OSH y $3 \%$ en transexuales). En el grupo de personas transexuales hubo una persona que poseía peces ornamentales, especie que no fue reportada en el grupo de OSH. Entre las personas que poseían varias especies de animales de compañía, el denominador común siempre lo constituyó el perro, que resultó la especie preferida para el $68 \%$ de ellas.

No se dispone de estudios similares en La Habana; sin embargo, en estudios realizados en otras provincias cubanas se encontró una mayor preferencia para perros y gatos (Beovides et al., 2011; L. Perdomo, datos no publicados).

La mayor parte de las personas involucradas en el estudio tenían una convivencia de 10 o más años con animales de compañía (55\% en cada grupo). Asimismo, 
Cuadro 3. Frecuencia (\%) de tenencia y preferencia de animales de compañía por personas con orientación sexual homoerótica (expresado en porcentaje)

\begin{tabular}{lcccccc}
\hline \multirow{2}{*}{$\begin{array}{l}\text { Animal de } \\
\text { compañía }\end{array}$} & \multicolumn{3}{c}{ Animal de tenencia } & \multicolumn{3}{c}{ Animal preferido } \\
\cline { 2 - 7 } & $\begin{array}{c}\text { Varones } \\
(\mathrm{n}=30)\end{array}$ & $\begin{array}{c}\text { Mujeres } \\
(\mathrm{n}=10)\end{array}$ & $\begin{array}{c}\text { Total } \\
(\mathrm{n}=40)\end{array}$ & $\begin{array}{c}\text { Varones } \\
(\mathrm{n}=30)\end{array}$ & $\begin{array}{c}\text { Mujeres } \\
(\mathrm{n}=10)\end{array}$ & $\begin{array}{c}\text { Total } \\
(\mathrm{n}=40)\end{array}$ \\
\hline Perro & 60 & 60 & 60 & 70 & 60 & 68 \\
Gato & 17 & 20 & 17 & 17 & 20 & 17 \\
Aves ornamentales & 13 & 10 & 12 & 7 & 10 & 8 \\
Peces ornamentales & - & - & - & - & - & - \\
Tortugas & - & - & - & 3 & - & 2 \\
Roedores & - & 10 & 3 & 3 & 10 & 5 \\
Otros & - & - & - & - & - & - \\
Varias especies & 10 & - & 8 & & & \\
\hline
\end{tabular}

Cuadro 4. Frecuencia (\%) de tenencia y preferencia de animales de compañía de 20 personas transe xuales

\begin{tabular}{lcc}
\hline $\begin{array}{l}\text { Animal de } \\
\text { compañía }\end{array}$ & $\begin{array}{c}\text { Animal de } \\
\text { tenencia }\end{array}$ & $\begin{array}{c}\text { Animal } \\
\text { preferido }\end{array}$ \\
\hline Perro & 45 & 60 \\
Gato & 30 & 15 \\
Aves & 10 & 10 \\
ornamentales & & \\
Peces & 5 & 5 \\
ornamentales & - & 5 \\
Tortugas & 10 & 5 \\
Roedores & - & - \\
Otros & 43 & \\
Varias especies & & \\
\hline
\end{tabular}

las respuestas indicaron una alta valoración sobre la satisfacción que sienten por convivir con sus animales ( 83 a $85 \%$ ) (cuadros 5 y 6 ). Las respuestas acerca de la interrelación entre dueños y animales de compañía fueron muy positivas, sobre todo, con relación a la salud psicoemocional.
Todos los varones del grupo OSH afirmaron que, gracias a la tenencia de sus animales, cuidaban más de su salud, ya que sin ellos sentían que podrían quedar desprotegidos; además, consideraban que les brindaban bienestar (93\%) y compañía $(90 \%)$. Las mujeres de este grupo, por otro lado indicaron que sus animales les brindaban bienestar y les servían de aliciente para cuidar más de su salud (90\%), y les servían de compañía (80\%) (Cuadro 5). Asimismo, el grupo de transexuales indicó que sus animales les brindaban bienestar (95\%), les incentivaban a cuidar su salud $(90 \%)$ y les servían de compañía (85\%) (Cuadro 6).

Sobre el particular, Gerzovich (1998) detalló los beneficios que reciben las personas al convivir en sus hogares con esta clase de animales y enfatizó sobre el hecho de que los perros facilitan los vínculos entre las personas y a la unión familiar y ayudan a liberarse del estrés, entre otros beneficios, lo cual se corrobora con las respuestas obtenidas en este estudio. Asimismo, Elton (2003) señala que el vínculo que el hombre ha creado con los animales de compañía va más allá de una necesidad práctica y llega a convertirse en una necesidad más bien emocional, similar a los resultados de este estudio. 
Cuadro 5. Tiempo de tenencia, satisfacción y efecto de la relación dueño-animal, expresado en porcentaje, de personas con orientación sexual homoerótica

\begin{tabular}{lccc}
\hline Característica & $\begin{array}{c}\text { Varones } \\
(\mathrm{n}=30)\end{array}$ & $\begin{array}{c}\text { Mujeres } \\
(\mathrm{n}=10)\end{array}$ & $\begin{array}{c}\text { Total } \\
(\mathrm{n}=40)\end{array}$ \\
\hline Tiempo de la tenencia (años) & 3 & 10 & 5 \\
$>1$ & 3 & 20 & 8 \\
$1-3$ & 10 & 10 & 10 \\
$4-6$ & 23 & 20 & 22 \\
$7-9$ & 60 & 40 & 55 \\
$>9$ & & & \\
& 83 & 80 & 83 \\
Satisfacción con la tenencia & 17 & 20 & 17 \\
Muy satisfecho & & & \\
Satisfecho & 90 & 80 & 88 \\
Efecto de la relación con el animal & 93 & 90 & 93 \\
Brinda compañía & 83 & 70 & 82 \\
Brinda bienestar & 67 & 40 & 60 \\
Libera tensiones y estrés & 60 & 50 & 57 \\
Satisface contacto & 67 & 50 & 62 \\
Mejora mi ánimo & 93 & 70 & 87 \\
Me comunico con él & 100 & 90 & 98 \\
Medio de comunicación familiar & 73 & 60 & 70 \\
Cuido mejor de mi salud & & & \\
Compañero para mi soledad &
\end{tabular}

Cuadro 7. Evaluación de algunos aspectos sobre los beneficios para la salud psicoemocional relacionados con la tenencia de animales de compañía en las personas con orientación sexual homoerótica $(\mathrm{n}=40)$

\begin{tabular}{|c|c|c|c|c|}
\hline \multirow{2}{*}{ Características } & \multicolumn{3}{|c|}{$\begin{array}{c}\text { Personas con orientación sexual } \\
\text { homoerótica }\end{array}$} & \multirow{2}{*}{$\begin{array}{c}\text { Transexuales } \\
\text { Total } \\
(\mathrm{n}=20)\end{array}$} \\
\hline & $\begin{array}{l}\text { Varones } \\
(\mathrm{n}=30)\end{array}$ & $\begin{array}{c}\text { Mujeres } \\
(\mathrm{n}=10)\end{array}$ & $\begin{array}{l}\text { Total } \\
(\mathrm{n}=40)\end{array}$ & \\
\hline \multicolumn{5}{|c|}{ Beneficios relación dueño /animal } \\
\hline Muy beneficiosa & 87 & 70 & 83 & 90 \\
\hline Beneficiosa & 13 & 30 & 17 & 10 \\
\hline \multicolumn{5}{|c|}{ Recomendar tenencia de animales } \\
\hline Sí & 80 & 80 & 80 & 100 \\
\hline No & 20 & 20 & 20 & 0 \\
\hline \multicolumn{5}{|c|}{ Animal de compañía/calidad de vida } \\
\hline Sí & 100 & 100 & 100 & 100 \\
\hline No & 0 & 0 & 0 & 0 \\
\hline
\end{tabular}


Cuadro 6. Tiempo de tenencia, satisfacción y efecto de la relación dueño-animal, expresado en porcentaje, de personas transexuales $(\mathrm{n}=20)$

\begin{tabular}{lc}
\hline Característica & $\%$ \\
\hline Tiempo de la tenencia (años) & \\
$>1$ & 5 \\
$1-3$ & 10 \\
$4-6$ & 10 \\
$7-9$ & 20 \\
$>9$ & 55 \\
Satisfacción con la tenencia & \\
Muy satisfecho & 85 \\
Satisfecho & 15 \\
Efecto de la relación con el animal & \\
Brinda compañía & 85 \\
Brinda bienestar & 95 \\
Libera tensiones y estrés & 80 \\
Satisface contacto & 50 \\
Mejora mi ánimo & 75 \\
Me comunico con él & 65 \\
Medio de comunicación & 60 \\
familiar & \\
Cuido mejor de mi salud & 90 \\
Compañero para mi soledad & 80 \\
\hline
\end{tabular}

\section{Beneficios para la Salud Psicoemocional}

En el Cuadro 7 se presentan los beneficios expresados por los encuestados en la salud psicoemocional debido a la tenencia de animales de compañía. En general, tanto las personas del grupo OSH como del grupo transexual consideraron mayoritariamente esta relación como muy beneficiosa.

El $100 \%$ de los transexuales y el $80 \%$ de las personas del grupo OSH recomendaban la tenencia de animales de compañía. El $20 \%$ que no recomendó correspondió a personas adultas, y argumentaban que debía tenerse en cuenta las condiciones y preferencias de las personas, ya que no a todos les pueden gustar los animales dentro de sus casas. Asimismo, Chur-Hansen et al. (2008), en un estudio con personas ancianas, indicaron que la posible renuencia a tener animales era mayormente de índole emocional (relacionadas con la muerte del animal o su desamparo si el dueño fallecía, así como el hecho de tener familiares y, por lo tanto, no necesitar la compañía del animal) o pragmáticas (por no poder dedicarles tiempo, entre otras).

Por último, la pregunta referida a la interrelación sobre la calidad de vida de las personas y la tenencia de los animales de compañía fue respondida por el $100 \%$ de forma afirmativa. Esta respuesta es demostrativa de lo que significa para las personas que integraron este estudio el hecho de compartir sus vidas junto a estos animales.

\section{Literatura Citada}

1. Beovides LM, Silveira PE, Norman MO. 2011. Evaluación del cuidado de la salud de mascotas en el municipio Santa Clara, Cuba. En: VII Congreso Internacional de Ciencias Veterinarias. La Habana, Cuba.

2. Castro M. 2008. La atención integral a transexuales en Cuba y su inclusión en las políticas sociales. Sexología y Sociedad 14(37). [Internet]. Disponible en: http://revsexologiaysociedad.sld.cu/ index.php/sexologiaysociedad/issue/ view/51

3. Chur-Hansen A, Winefield $H$, Beckwith M. 2008. Reasons given by elderly men and women for not owning a pet, and the implications for clinical practice and research. J Health Psychol 13: 988-995. doi: 10.1177/13591053 08097961

4. Elton ME. 2003. Diseño de una pauta de trabajo para la aplicación de terapia asistida con perros. Tesis de Médico Veterinario. Valdivia: Universidad Austral de Chile. 91 p. 
5. Gerzovich. C. 1998. Nuestro perro: uno más en la familia. Buenos Aires: Ed Planeta. $104 \mathrm{p}$.

6. Gómez LF, Atehortúa CG, Orozco SC. 2007. La influencia de las mascotas en la vida humana. Rev Col Cienc Pec 20: 377-386.

7. Jofré LM. 2005. Visita terapéutica de mascotas en hospitales. Rev Chil Infectol 22: 257-263. doi: 10.4067/S071610182005000300007

8. McConnellAR, Brown CM, Shoda TM, Stayton LE, Martin CE. 2011. Friends with benefits: on the positive consequences of pet ownership. J Pers Soc Psychol 101: 1239-1252. doi: 10.1037/a0024506

9. Palú A, Orozco MI, García A, Pérez IM, Miranda S. 2010. Aportes de la epidemiología al estudio de la diversidad sexual masculina. Medisan 14: 217-224.

10. Scourfield J, Roen K, McDermott E. 2008. Lesbian, gay, bisexual and transgender young people's experience of distress: resilience, ambivalence and self-destructive behaviour. Health Soc Care Community 16: 329-336. doi: 10.1111/j.1365-2524.2008.00769.x 